\title{
A Cognitive Task Analysis of Using Pictures To Support Pre-Algebraic Reasoning
}

\author{
Kenneth R. Koedinger (koedinger@cmu.edu) \\ Human-computer Interaction Institute, Carnegie Mellon University \\ 5000 Forbes Ave. Pittsburgh, PA 15213-3890 \\ Atsushi Terao (atsushi@ cs.cmu.edu) \\ Human-Computer Interaction Institute, Carnegie Mellon University \\ 5000 Forbes Ave. Pittsburgh, PA 15213-3890 USA
}

\begin{abstract}
We present an analysis of hypothesized advantages of pictorial representations for improving learning and understanding of pre-algebraic quantitative reasoning. We discuss a "Picture Algebra" strategy that has been used successfully by $6^{\text {th }}$ grade students as part of a new middle school mathematics curriculum. This strategy supports students in sense making both as they construct pictorial representations and as they use them to cue appropriate computations. Although we demonstrate that $6^{\text {th }}$ grade students can use this strategy to successfully solve algebra-level problems, our detailed production rule analysis revealed limitations in our instructional approach and targeted areas for improvement.
\end{abstract}

\section{Introduction}

As part of a larger effort to develop a $6^{\text {th }}$ grade mathematics course including both a textbook and Cognitive Tutor software (cf., Koedinger, Anderson, Hadley, \& Mark, 1997), we have been exploring the use of pictorial representations to support student reasoning and learning (Rittle-Johnson \& Koedinger, 2001). Here we investigate the claim that pictorial representations can help students gain early entry into algebraic reasoning and build a foundation that will facilitate more effective learning of formal algebra.

Why might using pictures or diagrams be advantageous? Cognitive scientists have presented arguments and experiments for the advantages of diagrams (e.g., Cheng, 1999; Larkin \& Simon, 1987). According to Larkin and Simon (p. 98), "a diagram can be superior to a verbal description for solving problems" for three reasons. First, diagrams reduce problem-solving search by providing localized groupings of relevant information. Second, diagrams reduce the need for matching symbolic labels. Third, diagrams support perceptual inferences that are often easier than corresponding symbolic inferences.

Others have presented arguments for the use of diagrams for mathematics instruction in particular. The mathematics standards of the National Council of Teachers of Mathematics (NCTM, 2000) recommends use of pictures to support students in developing a conceptual understanding of mathematics. Pictorial representations are used extensively in Asian curricula (cf., Singapore Ministry, 1999). This usage may be a factor in the success of Asian countries on international mathematics assessments (TIMSS, 1996).

Despite these arguments for the advantages of pictures, there is also reason for caution. One argument for the use of alternative representations, like pictures, is that traditional instruction focuses too much on errorprone rote learning. However, students may also acquire rote procedures when learning to use alternative representations. Further, learning an alternative representation takes time that might be better spent learning the standard representation.

In this paper we introduce the "Picture Algebra" strategy, present student data on the use of it, and discuss a production rule model of the strategy and implications for transfer and instructional design.

\section{Picture Algebra}

Try to solve the Cans problem shown in Table 1 and reflect on the strategy that you use to do so. We have informally observed that many adults do not directly infer what arithmetic operations are needed to solve this problem. Instead, most begin by translating the problem statement to one or more algebraic equations, for instance, $x+(x+9)+(x+17)=227$. They then perform transformations on the equation to arrive at a solution. Although a few use other means (cf., Hall et al., 1989), most find this problem difficult without the use of algebraic equations. In other words, this is arguably an "algebra problem" that we might expect to be out of reach of students without algebra instruction, for instance, $6^{\text {th }}$ graders.

Figure 1A shows a $6^{\text {th }}$ grade student's solution to this problem using a "Picture Algebra" strategy that was taught to students as part of our middle school mathematics curriculum. Like other problem-solving strategies, Picture Algebra can be described in two phases: a representation phase and a solution phase. In the representation phase, the student first translates the 
Table 1. Three Problems Solved by $6^{\text {th }}$ Graders

Cans: The sixth, seventh and eight grade classes brought in canned goods for the needy. They collected 227 cans between the grades. Sixth grade collected 9 more cans than eighth grade, and seventh grade collected 17 more cans than the 8th grade. How many cans did each grade collect?

Beanie: Robin has 6 fewer beanie babies than Angie. If they have 42 beanie babies altogether, how many beanie babies does Robin have?

CD: Carissa wants to buy the latest Out o' Sync CD. She also wants to buy a magazine and a poster about her favorite band. The magazine costs $\$ 8$ less than $\mathrm{CD}$, and the poster costs $\$ 12$ less than CD. The total cost for all three items is $\$ 46$. How much does each item cost?

phrase "sixth grade collected 9 more cans than eighth grade" into a box diagram by drawing a box to represent the cans collected by the $8^{\text {th }}$ grade and a larger box to represent the cans collected by the $6^{\text {th }}$ grade. The larger box is made up of two smaller boxes, one that is the same size as the $8^{\text {th }}$ grade box and one that represents the 9 more cans the $6^{\text {th }}$ grade collected. Similarly, the student draws two boxes to represent the $7^{\text {th }}$ grade cans. In both cases, the extra box off to the right is labeled with the given "more-than" values, 9 and 17. In the final step of the representation phase, the student represents the total number of cans, 227, by drawing a bracket to the right of all the boxes.

This picture representation and the algebra equation, $x+(x+9)+(x+17)=227$, are "informationally equivalent" in Larkin \& Simon's terms. The three equal sized boxes on the left are analogous to the three x's in the equation, the extra boxes represent the +9 and + 17 , and the bracket and the 227 represent the equal sign and right hand side of the equation. Despite this similarity, the picture and equation representations are not "computationally equivalent. Whereas the equation is a 1D "horizontal" representation, the picture is $2 \mathrm{D}$ and takes advantage of both the horizontal and vertical dimensions to better support the inferences needed to solve the problem.

As we step through the Picture Algebra solution phase, notice how inferences are visually supported in a way they are not in the analogous equation solving steps. The first step in the solution phase is to parse or comprehend the representation, picture or equation. Past research has shown that both equation comprehension (Payne \& Squibb, 1990) and equation production (Heffernan \& Koedinger, 1998) are particularly difficult sets of skills for students to acquire. For instance, students must learn when they can and cannot rearrange elements of an equation. Although experts know they can in effect ignore the parentheses in " $x+(x+9)+(x+17) "$ and transform the expression into " $x+x+x+9+17$ " and then " $3 x+$ 26 ", the steps involved are non-obvious to students and prone to error. In contrast, the analogous steps in the
Picture Algebra strategy are visually supported. The vertical organization of the bars makes it more apparent that the three equal unknown boxes can be grouped separately from the two extra boxes for 9 and 17. As a consequence, students can "see" that if they subtract these extra boxes, they will be left with the three equal boxes. Notice the arithmetic on the left in Figure 1A where the student computes both $9+17=26$ and $227-$ $26=201$. This step is analogous to the "subtract from both sides" transformation in equation solving, however, it is more perceptually intuitive as students can visualize chopping off the extra boxes. Next the student divides 201 by 3 (see the bottom middle of Figure 1A) to get the value, 67, of the three equal boxes. Finally, the student adds back the 9 and 17 to get the values of cans collected by the $6^{\text {th }}$ and $7^{\text {th }}$ grade.

\section{Student Solution Data}

We analyzed 35 sixth graders' solutions to the three problems shown Table 1. The Cans and Beanie problems were given as part of a unit test. To get a sense for whether the representation or solution phase of Picture Algebra is more difficult for students, we provided a correct picture representation for some students but not for others. Half the students were given a test in which a diagram was given for the Cans problem but not for the Beanie problem. The remaining students were given a test in which a diagram was given for the Beanie problem, but not the Cans problem. Students solved the CD problem (with no diagram provided) as part of a warm-up activity on a later day. (One student was absent on each day.)

The Cans problem involves three unknown quantities (cans collected by $6^{\text {th }} 7^{\text {th }}$, and $8^{\text {th }}$ grades) and more-than relations between these quantities. Students were initially instructed on Picture Algebra with simpler two quantity more-than problems. The Beanie problem also has two quantities but is more difficult because of the less-than (or "fewer") relation between them (Lewis, 1989). The CD problem combines both dimensions of difficulty as it involves three quantities and less-than relations. One question of interest is whether these dimensions of difficulty are independent.

\section{Overall Performance Results}

The first result of note is that $6^{\text {th }}$ graders can effectively use pictorial representations to solve "algebraic" problems. They were $68 \%$ correct on both the Cans and Beanie problems and $32 \%$ correct on the CD problem. Such problems are challenging for many older students. For instance, Bednarz \& Janvier (1996, p. 120) found that pre-algebra students (same age as US $7^{\text {th }}$ graders) were only $5 \%$ correct on the following problem:

380 students are registered in sports activities for the season. Basketball has 76 more students than skating and swimming has 114 more than basketball. How many 


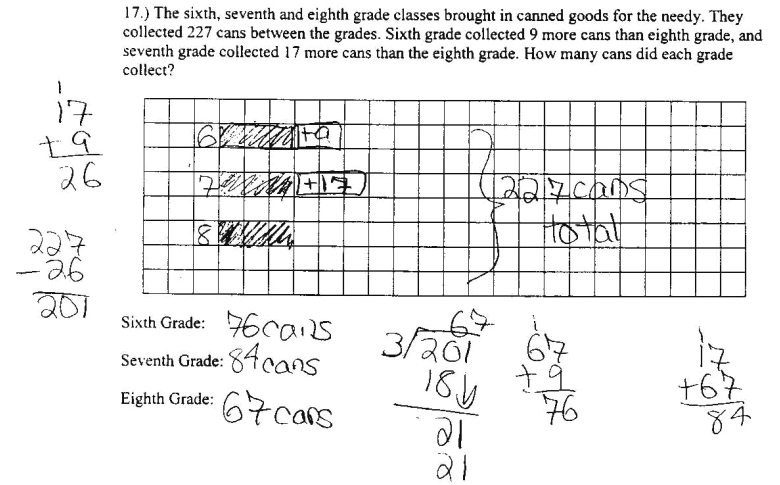

(A) Correct size-preserving picture for Cans problem

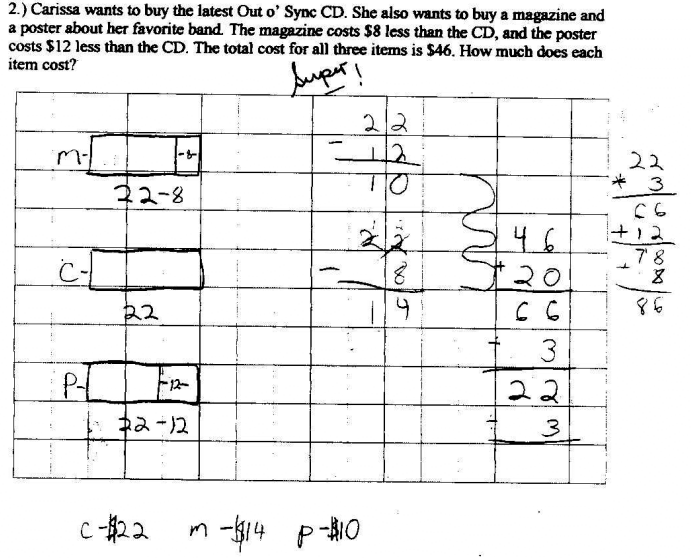

(B) Correct size-preserving picture for $\mathrm{CD}$ problem

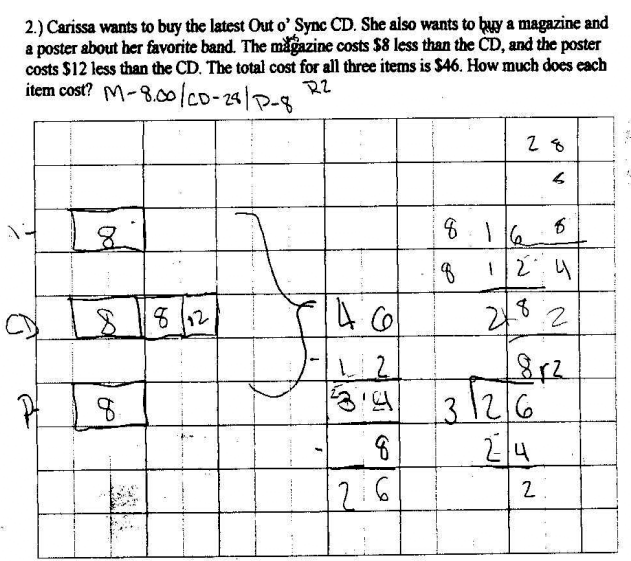

(C) Wrong picture for CD problem

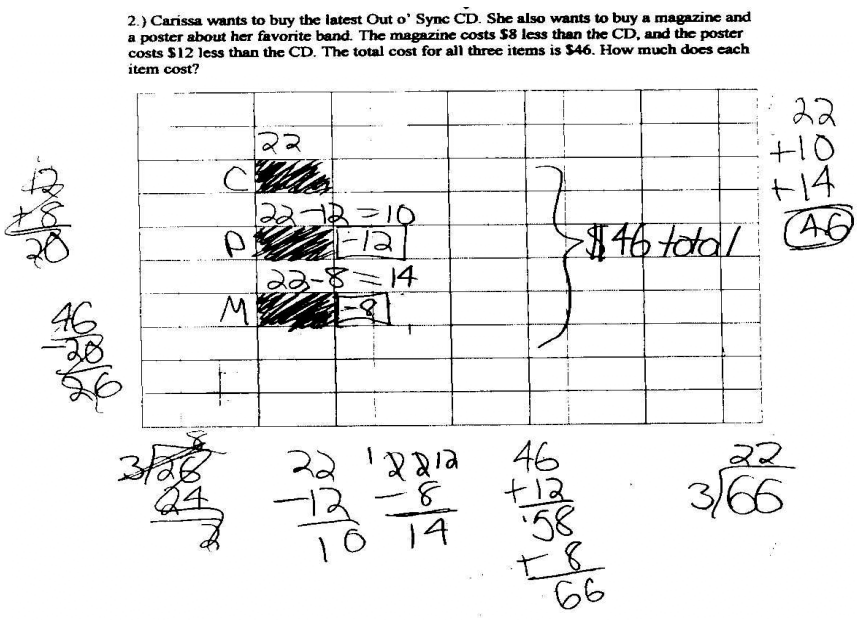

(D) correct abstract picture for $\mathrm{CD}$ problem

Figure 1. Correct and incorrect $6^{\text {th }}$ grade students' Picture Algebra solutions.

students are there in each of the activities?

As another reference point, Koedinger \& Alibali (1999) collected data on college students' performance on the following relatively simple problem:

There are 38 students in class. If there are 6 more girls than boys, how many boys are in the class?

Students in a college algebra course were only $54 \%$ correct and even CMU students with a mean math SAT of 719 were $86 \%$ correct.

Students given a picture on the Cans and Beanie problems were $71 \%$ correct in both cases and were $65 \%$ correct on both without the picture. That being given the picture did not help much on these problems indicates that the representation phase was relatively easy for students. In contrast, the representation phase on the CD problem was not easy. Students drew a good picture in only $32 \%$ of CD solutions, but were $64 \%$ correct when they did. They were only $17 \%$ correct when a picture was missing or incorrect.
We were surprised at students relatively poor performance on the CD problem $(32 \%)$ given that two key sources of difficulty in that problem, 3 quantities and less-than relations, were each present individually in either the Cans problem (3 quantities) or the Beanie problem (less-than relation). A simple surface-level analysis of problem difficulty factors would predict that students would be, at worst, $42 \%$ correct (.65*.65) on the CD problem. Clearly, such a surface level analysis is insufficient to understand why the CD problem was so hard for students. Our production rule analysis below provides a better explanation.

\section{Diagramming Strategies}

We classified the pictures students drew into 5 categories: size-preserving, incomplete, wrong, abstract, and no-diagram. In a "size-preserving" picture, all quantities, or parts of quantities, are represented by boxes whose sizes correspond with the 
relative sizes of the quantities. That is, bigger quantities are represented by bigger boxes. Unknown quantities can be any size, but equal quantities must be represented by equal size boxes. All quantities are represented and all known quantities or parts of quantities are labeled with their given value. Figures 1A-B show examples of "size-preserving" pictures. An "incomplete" picture was one that was otherwise correct, but was missing quantities or labels. A "wrong" picture has boxes that are the wrong relative sizes. Figure 1C shows an example where the box for the magazine cost (top box) is the same size as the box for the poster cost (bottom box) when, in fact, the magazine cost is bigger than the poster cost.

An "abstract" picture is shown in Figure 1D. The boxes in Figure 1D violate the size preserving constraint. Whereas the CD cost is largest, it appears as the smallest box (the top one). However, some students correctly interpreted these extra boxes (labeled -12 and -8) as, in essence, having a negative size.

A potential advantage of the abstract picture is that it may incrementally move students toward the kinds of abstract inferences needed for symbolic algebra transformations. On the easier Cans and Beanie problems, the 7 solutions employing an abstract picture were all correct. However, one disadvantage of the abstract picture is that without the size-preserving cues, students can easily confuse the operations that need to be performed and, for instance, subtract when they should add. This confusion led 4 of the 10 students who used an abstract picture on the CD problem to an incorrect answer. Further, even among the 6 students whose abstract picture led to a correct solution, there is evidence of confusion. Notice the arithmetic on the left in Figure 1D where the student incorrectly subtracts 20 from 46 rather than adding 20 and 46. The student abandons this approach when he notices that 3 does not divide evenly into 26 . This correct solution may not have come from understanding, but from a shallow school heuristic that problems usually come out even.

\section{Production Rule Model of Picture Algebra}

We performed a production-rule analysis of typical correct and incorrect student solutions to the three problems. Table 2 shows examples of key productions.

\section{Production Trace of Cans Solution}

The correct solution to the Cans problem with a sizepreserving diagram (see Figure 1A) is traced with 13 productions. These productions are the same ones needed to solve a more-than problem dealing with two unknown quantities and thus we would expect good transfer from class instruction. To process 3 unknown quantities rather than 2, some productions are executed multiple times. When drawing a size-preserving picture the model first has to draw a "base" box (P2a in Table
Table 2. Picture Algebra Production Rule Examples

P0a: Change-less-than-to-more-than

If goal is to draw boxes to represent $=\mathrm{Q} 1$ is $=\mathrm{X}$ less than $=\mathrm{Q} 2$

then set goal to draw boxes to represent $=\mathrm{Q} 2$ is $=\mathrm{X}$ more than $=\mathrm{Q} 1$

P0b: Change-less-than-to-more-than-negative

If goal is to draw boxes to represent $=\mathrm{Q} 1$ is $=\mathrm{X}$ less than $=\mathrm{Q} 2$,

then set goal to draw boxes to represent $=\mathrm{Q} 1$ is minus $=\mathrm{X}$ more than $=\mathrm{Q} 2$

P2a: Draw-base-box-more-than

If goal is to draw boxes to represent $=\mathrm{Q} 2$ is $=\mathrm{X}$ more than $=\mathrm{Q} 1$ and no box has been drawn

then draw a first box for $=\mathrm{Q} 1$

P3c: Draw-comp-box-equal-part-repair

If goal is to draw boxes to represent $=\mathrm{Q} 2$ is $=\mathrm{Y}$ more than $=\mathrm{Q} 3$

and first box for $=\mathrm{Q} 2$ with the size $=\mathrm{S} 1$ is drawn and no box for $=\mathrm{Q} 3$ has been drawn

then draw a first box for $=\mathrm{Q} 3$ with the size $=\mathrm{S} 1$

P6a: Check-equal-size-boxes-with-extra

If goal is to solve a problem with a box diagram and there are equal-size boxes and the value of an extra box for $=\mathrm{Q} 1$ is $=\mathrm{X}$ and the box with value $=\mathrm{X}$ has not been removed then set goal to remove the extra box

2 ). This box represents the $8^{\text {th }}$ grade cans. Other productions draw "equal-part" boxes for the equal parts of the $6^{\text {th }}$ and $7^{\text {th }}$ cans and "extra-part" boxes for the 9 and 17 . The final production in the representation phase draws and labels the bracket with the total 227.

At this point, the solution phase begins with production $\mathrm{P} 6 \mathrm{a}$, which sets a goal to remove an extra box. This production fires twice in the Cans solution, once with $=\mathrm{Q} 1$ set to $6^{\text {th }}$ grade cans and $=\mathrm{X}$ to 9 and $\mathrm{a}$ second time with $=\mathrm{Q} 1$ set to $7^{\text {th }}$ grade cans and $=\mathrm{X}$ to 17. Once all quantities represented as extra boxes (i.e., 9 and 17) are subtracted from the total quantity (227), further productions find the base quantity by dividing the new total (201) by the number of equal-size boxes (3). The model finishes with productions for finding the other two quantities by adding the extra box values (9 and 17) to the value of the base quantity (67).

\section{Production Trace of Beanie Solution}

The key to the model's solution of the Beanie problem is production $\mathrm{POa}$ that converts a less-than relation to a more-than relation. Once the model sees "Robin has 6 fewer beanie babies than Angie" as "Angie has 6 more beanie babies than Robin", it can solve the problem just as it would a more-than problem. Students' relatively good performance on the Cans and Beanie problems is consistent with a production rule transfer analysis (Singley \& Anderson, 1989). Relative to the familiar two-quantity, more-than problems students were instructed on, only repetitions of already known 
productions are needed for the Cans problem and only one new production is needed for the Beanie problem.

\section{Production Traces of CD Solutions}

It turns out the simple less-than to more-than transformation performed by $\mathrm{P} 0 \mathrm{a}$ does not work so well for solving the $\mathrm{CD}$ problem. A production rule trace of the incorrect solution to the CD problem in Figure $1 \mathrm{C}$ illustrates this point. As in the Beanie trace, the model begins by firing P0a to convert "price of magazine is $\$ 8$ less than the price of $C D "$ to "price of $\mathrm{CD}$ is $\$ 8$ more than the price of magazine". It then draws the single smaller box for the magazine cost (top of Figure $1 \mathrm{C}$, but without 8 inside) and the equal-part and extrapart boxes for the CD cost (first two boxes in the CD row, without the 12 box or the first 8 label). The representation is correct at this point, but after converting "the price of poster is $\$ 12$ less than the price of CD" relation to "the price of $\mathrm{CD}$ is $\$ 12$ more than the price of poster" the model has trouble.

If the model only had the productions needed for the Cans and the Beanie problem, it would reach an impasse here. Usually the smaller base of the morethan statement (Poster in this case) has already been drawn and there are productions for drawing the equaland extra-parts of the larger quantity (CD). However, in this case, it is the smaller box (Poster) that has not been drawn. Our model predicts that students reach an impasse at this point and must implement a "repair" (VanLehn, 1983). The production P3c in Table 2 represents a result of this repair. It draws a base box for the Poster cost that is the same size as the equalpart of the CD cost. Next, an over-general production adds an extra box to the CD row for the "12 more". This production is over-general because it is missing a constraint to check that an extra box has not already been drawn (the middle 8 box in the CD row). The model continues, like the student, to correctly compute values consistent with this incorrect representation of the problem.

With only one production difference from the Cans and Beanie problems this solution is a relatively easy transfer. This provides an explanation for the frequency of this odd incorrect solution strategy. 8 of 34 students drew pictures for the $\mathrm{CD}$ problem like the one shown in Figure $1 \mathrm{C}$ with two extra boxes in the CD row.

Students found two ways to be successful on the CD problem. Figure 1D shows an example of one of these ways. The model again starts by converting the given less-than relations to more-than relations, but not by changing the position of the quantities in the relation, but by negating the difference value (P0b in Table 2). For example, "price of magazine is $\$ 8$ less than the price of CD" is converted into "price of magazine is $\$ 8$ more than the price of CD". The model then draws an abstract diagram with an extra box in the Magazine row that has a negative value. New productions are needed to deal with extra boxes with negative values. One of these productions removes an extra negative box by adding the absolute value of the box to the total $(46+12$ and +8$)$. A similar production is needed in the final steps of the solution to combine these negative values with the unknown value (22).

A second approach to the CD problem involves directly representing the less-than relations in the picture (see Figure 1B). In this approach, the larger CD cost remains the base quantity in both relations. That the magazine cost is $\$ 8$ less is represented by labeling the space between the right end of the Magazine bar and the right end of the Poster bar. (It would be better if the student had not put a box around the 8 as this box implies the size of the Magazine cost includes this 8 when it does not.) Only one student on the CD problem drew a picture that was close to size preserving.

Both of the correct solution strategies for the CD problem involve more new productions (4 and 7) than the incorrect solution strategy (1). While the abstract picture strategy involves fewer new productions (4) than the size-preserving strategy (7), there are other relative advantages of the size-preserving strategy.

The new productions required for the size-preserving strategy are mostly straightforward analogies with existing productions, where less-than is substituted for more-than. More importantly, this strategy provides more reliable perceptual support for inferences. To get three equal bars, one must fill in the empty space to the right of the Magazine and Poster boxes (see Figure 1B). This "filling in" is a perceptual cue for addition. In contrast, in the abstract picture (see Figure 1D), the perceptual cues suggest "removing" or subtraction and, as we saw, many students fell for or were distracted by this cue. To succeed with this strategy, students have to do explicit symbolic processing to infer that taking away a negative is the same as adding.

An important consequence of this analysis was the recognition that students should be instructed not to draw boxes around extra missing parts (see the - 8 - and - 12 - in Figure 1C), but to use a double-headed arrow or bracket to mark the empty space.

\section{Discussion}

The Picture Algebra strategy benefits from a number of positive features of diagrammatic representations: grouping to facilitate search, reducing the need for symbolic labels, and substituting intuitive perceptual inferences for more difficult logical inferences (Larkin $\&$ Simon, 1987). The potential value of this strategy is evidenced by the observed success of $6^{\text {th }}$ grade students on algebra-level problems that are quite difficult for many older students.

Teachers always encourage students to check their answers and students appear more likely do so when 
using Picture Algebra (Fig 1B,C,D) than when using other strategies. We speculate that by evoking students' spatial intuitions, the pictorial representation puts students in a "sense making" mode that leads to greater self-monitoring (cf., Kalchman, Moss, \& Case, 2001).

However, instruction based on pictures is not a panacea. Although pictures may facilitate students' reasoning and learning, it is not trivial for students to learn to use such representations flexibly and with understanding. Not all students using Picture Algebra engaged in sense making. Some made errors typical of equation solving (e.g., subtracting when they should add) and failed to catch them even with the visual support of the picture (cf., Lewis, 1989).

Although students performed relatively well on problem demands presented individually in the Cans and Beanie problems, they had much greater trouble with the CD problem that combined both demands. Our production rule analysis provides a detailed explanation for why this problem is significantly more difficult. The difficulty arises from the order in which the quantities are related to each other and potential traps from inappropriately selecting a "base" quantity.

A novel prediction of our model is that a 3-quantity more-than problem of the form "A is $\mathrm{X}$ more than $\mathrm{B}$ and $\mathrm{A}$ is $\mathrm{Y}$ more than $\mathrm{C}$ " should be almost as difficult as the CD problem (a 3-quantity less-than problem). This prediction follows from the fact that the model essentially converts the CD problem into this form. Similarly, the model predicts that a 3-quantity less-than problem of the form "A is $\mathrm{X}$ less than $\mathrm{B}$ and $\mathrm{A}$ is $\mathrm{Y}$ less than $C^{\prime \prime}$ should actually be relatively easy, more like the Cans problem. The generation of such predictions has pushed us to rethink and improve our curriculum design to better address the previously hidden skills revealed by our production rule analysis.

This paper reports on theoretical and empirical steps toward answering the following research questions:

1. Can instruction on the use of Picture Algebra help younger students gain entry into algebraic reasoning sooner than direct instruction on formal algebra?

2. Can instruction on Picture Algebra help younger students build a foundation that will improve and accelerate later learning of formal algebra?

Because of the complexity and cost of performing classroom experiments that could directly address these questions, these questions are excellent candidates to test the applicability of cognitive theory. Our goal is to employ cognitive theory and lower cost empirical studies to provide strong arguments for and/or against these claims. With such arguments in hand, we are better prepared to assess the potential benefit of a costly classroom experiment and, perhaps more importantly, facilitate the design of an instructional method that is most likely to be successful.

\section{Acknowledgments}

This work was supported by NSF ROLE grant REC0087396 and a curriculum development grant from Carnegie Learning, Inc. (www.carnegielearning.com).

\section{References}

Bednarz, N., \& Janvier, B. (1996). Emergence and development of algebra as a problem-solving tool. In Bednarz, Carolyn, \& Lee (Eds.), Approaches to Algebra. (pp. 115-145). Dordrecht, Netherlands: Kluwer.

Cheng, P. C.-H. (1999). Interactive law encoding diagrams for learning and instruction. Learning and Instruction. 9(4), 309-326.

Hall, R., Kibler, D., Wenger, E., \& Truxaw, C. (1989). Exploring the episodic structure of algebra story problem solving. Cognition and Instruction, 6, 223-283.

Heffernan, N.T. \& Koedinger, K. R. (1998). A developmental model for algebra symbolization. In Proceedings of the Twentieth Annual Conference of the Cognitive Science Society, (pp. 484-489). NJ: Erlbaum.

Kalchman, M., Moss, J. \& Case, R. (2001). Psychological models for development of mathematical understanding. In S. Carver \& D. Klahr (Eds.), Cognition and instruction: Twenty-five years of progress (pp. 1-38). NJ: Erlbaum.

Koedinger, K. R. \& Alibali, M. W. (1999). A developmental model of algebra problem solving. Annual meeting of the American Educational Research Association.

Koedinger, K. R., Anderson, J. R., Hadley, W. H., \& Mark, M. A. (1997). Intelligent tutoring goes to school in the big city. International Journal of Artificial Intelligence in Education, 8, 30-43.

Larkin, J. H., \& Simon, H. A. (1987). Why a diagram is (sometimes) worth ten thousand words. Cognitive Science, 11, 65-99.

Lewis, A. B. (1989). Training students to represent arithmetic word problems. Journal of Educational Psychology, 81, 521-531.

National Council of Teachers of Mathematics (2000). Principles and Standards for School Mathematics. ISBN 0-87353-480-8. Reston, VA: NCTM.

Payne \& Squibb (1990). Algebra mal-rules and cognitive accounts of error. Cognitive Science, 14, 445-491.

Rittle-Johnson, B. \& Koedinger, K. R. (2001). Using cognitive models to guide instructional design: The case of fraction division. In Proceedings of the Twenty-Third Annual Conference of the Cognitive Science Society, (pp. 857-862). NJ: Erlbaum.

Singapore Ministry of Education (1999). Primary mathematics 6A Third Edition. Singapore: Federal Publications. www.singaporemath.com.

Singley, M. K., \& Anderson, J. R. (1989). Transfer of Cognitive Skill. Hillsdale, NJ: Erlbaum.

TIMSS (1996). Third International Mathematics and Science Study. National Research Coordinators: National Center for Educational Statistics.

Van Lehn, K. (1983). On the representation of procedure in repair theory. In H. P. Ginsburg (Eds.), The development of mathematical thinking. New York: Academic Press 